\title{
Are renal tumour scoring systems better than clinical judgement at predicting partial nephrectomy complexity?
}

\author{
Ravi M. Kumar, MD'; Luke T. Lavallée, MD',2; Darren Desantis, MD'; Sonya Cnossen, MSc2; \\ Ranjeeta Mallick, PhD'; llias Cagiannos, MD'; Chris Morash, MD'; Rodney H. Breau, MD',2
}

Division of Urology, Department of Surgery, The Ottawa Hospital, University of Ottawa; ${ }^{2}$ Ottawa Hospital Research Institute; Ottawa, ON, Canada

Cite as: Can Urol Assoc J 2017;1 1 (6):199-203. htrp://dx.doi.org/10.5489/cuaj.4228

\section{Abstract}

Introduction: We aimed to determine how renal tumour scoring systems, such as RENAL, PADUA, and Centrality (C)-index, compare to clinical judgement at predicting time required for tumour removal and kidney reconstruction during partial nephrectomy.

Methods: A consecutive cohort of partial nephrectomy patients treated at The Ottawa Hospital, a tertiary care uro-oncological centre, was retrospectively reviewed. Preoperative axial images were reviewed by four experienced urological oncologists who independently rated the complexity of a partial nephrectomy from 1-10 to generate a clinical judgement score. Two independent reviewers determined the RENAL, PADUA, and C-index scores. The time to complete tumour resection and renal reconstruction during partial nephrectomy was prospectively recorded.

Results: During the study period, 104 partial nephrectomies were performed. The mean partial nephrectomy complexity score based on clinical judgement was 3.4 (standard deviation [SD] 2.1) out of 10 . There was good agreement between surgeons in assessing tumour complexity (intraclass correlation coefficient $0.72 ; 95 \%$ confidence interval $[\mathrm{CI}] 0.65,0.78)$. The mean RENAL score was 6.7 (SD 1.6) out of a maximum of 12, the mean PADUA score was 8.5 (SD 1.5) out of a maximum of 14, and the mean C-index score was 3.8 (SD 2). Mean resection and reconstruction time was 24 minutes (SD 10 minutes). The correlation between clinical judgement score and time was 0.27 ( $p=0.005)$. The correlation between renal tumour scoring systems and time was 0.20 $(p=0.04)$ for RENAL, $0.21(p=0.03)$ for $C$-index, and $0.26(p=0.007)$ for PADUA. RENAL and PADUA scores were significantly associated with surgical and total complications.

Conclusions: The majority of variance in ischemia time is not explained by clinical judgement or renal tumour scoring systems. Renal tumour scoring systems were not better than the clinical judgement of urological oncologists at predicting ischemia time during partial nephrectomy.

\section{Introduction}

In appropriately selected patients, partial nephrectomy is preferred compared to radical nephrectomy, since partial nephrectomy results in similar oncological outcomes and improved postoperative renal function. ${ }^{1}$ Partial nephrectomy is a more challenging operation with a well-described learning curve, and is associated with an increased risk of perioperative complications. ${ }^{2,3}$ Therefore, the optimal surgical approach should be based on balancing the benefits and risks for each patient.

Several scoring systems have been proposed to objectively quantify the difficulty of a partial nephrectomy using preoperative cross-sectional images. These scores are designed to facilitate cohort comparisons and help surgeons make clinical decisions by predicting surgical risk. The three most commonly cited scoring systems are: RENAL Nephrometry Score (RENAL), PADUA (PADUA), and Centrality (C)-index. ${ }^{4-6}$ RENAL and PADUA scores are divided into several components that combined provide a score based on tumour characteristics, such as radius, exophytic vs. endophytic nature, and nearness to the collecting system. The component scores are summed to obtain an overall score and risk group. C-index incorporates the distance from the tumour centre to the kidney centre and the tumour radius to assign a complexity score. Increased surgical complexity of a tumour is associated with higher RENAL and PADUA scores, and lower C-index scores. These scoring systems have been shown to correlate with treatment decisions, intraoperative ischemia time, and postoperative complications..$^{7-12}$

Despite the proven prognostic performance of renal tumour scoring systems, many surgeons may not use these systems in clinical practice because they are time-consuming and are not proven to be superior to clinical judgement. ${ }^{13}$ The purpose of this study was to determine if clinical judgement alone performs better or worse than renal tumour scoring systems at predicting ischemia time and postoperative complications for patients undergoing partial nephrectomy. 
Kumar et al.

\section{Methods}

Institutional ethics board approval was obtained to review a consecutive cohort of partial nephrectomy patients treated at The Ottawa Hospital. Patients were excluded if they had a pelvic kidney or a horseshoe kidney. The medical record for each patient was reviewed using a structured data abstraction template to determine patient age, sex, preoperative renal function, and presence of comorbid disease (diabetes, hypertension, smoking history, and heart disease). Operative characteristics, including surgical approach and estimated blood loss, were obtained from the medical record.

\section{Scoring systems}

Preoperative axial images were reviewed by two independent physicians to determine the RENAL, PADUA, and $\mathrm{C}$-index scores for each tumour. Disagreements in scores were decided by rereview and consensus. The RENAL score consists of tumour radius, exophytic/endophytic properties, nearness to the collecting system, anterior/posterior, and the location relative to the polar line. ${ }^{4}$ Apart from the anterior/posterior designation, each component is scored on a three-point scale. The minimum possible score is 3 and the maximum score is 12. PADUA score includes: tumour diameter, location relative to polar lines, anterior/posterior location, medial/lateral location, collecting system association, renal sinus association, and exophytic/endophytic extent. ${ }^{5}$ PADUA scores range from 6-14. C-index is measured using Pythagorean theorem to calculate the distance between the tumour centre and the kidney centre, and then dividing the quotient by the tumour radius. ${ }^{6} \mathrm{~A}$ lower $\mathrm{C}$-index score designates a more complex tumour location.

\section{Clinical judgement}

For each patient, a 10-second video of the preoperative axial images of the affected kidney was created. Each video was de-identified and distributed to four urological oncologists. The number of years in practice (since completing fellowship) was one, six, 11, and 18. All surgeons perform partial nephrectomy procedures. The urological oncologists were asked to independently scroll through the video as they would in a typical clinical setting and rate the surgical complexity of partial nephrectomy on a scale of 1-10. Surgeons were blinded to the renal tumour system scores and the responses of other raters. The score used for analysis was the average of the four raters complexity score.

\section{Outcomes}

The primary outcome was ischemia time, defined as the time required to complete tumour resection and renal reconstruction during partial nephrectomy. Ischemia time was prospectively recorded in the medical record. If renal hypothermia was used, the time used to cool the kidney was subtracted from the total ischemia time because no dissection is performed during this time.

The secondary outcome was postoperative complications within 30 days from surgery. Postoperative complications were collected using a priori definitions and the severity was categorized using the Clavien-Dindo classification system. ${ }^{14}$ Surgical complications included death, surgical re-exploration, conversion to open, bleeding necessitating transfusion, urine leak, dialysis, lymphocele, pyelonephritis, perinephric abscess, retroperitoneal hematoma, wound infection, urinary tract infection, pneumothorax, pseudoaneurysm, and bowel perforation. Total complications included surgical complications, as well as medical complications, including cardiovascular (new-onset hypertension, angina, myocardial infarction, new-onset arrhythmia, congestive heart failure), pulmonary (symptomatic atelectasis, pneumonia, respiratory failure), thromboembolic (deep vein thrombosis, pulmonary embolism, cerebrovascular accident), gastrointestinal (ileus, clostridium difficile infection, pancreatitis), and metabolic (hyperkalemia, rhabdomylosis). The expected rates of complications following partial nephrectomy have been previously described by Patel et al. ${ }^{15}$

\section{Statistical analysis}

For clinical judgement score, the level of agreement between the four evaluating surgeons was measured using the intraclass correlation coefficient (ICC). While there are no set thresholds for ICC, ranges of 0.0-0.2, 0.3-0.4, $0.5-0.7,0.7-0.8$, and $>0.8$ can be considered poor, fair, moderate, strong, and almost perfect agreement, respectively. To compare tumour scoring models to ischemia time, the coefficient of determination $\left(r^{2}\right)$ was calculated. The $r^{2}$ value indicates what proportion of the variability in outcome (ischemia time) is explained by the scoring system. Associations between clinical judgement or scoring systems and complications were determined using t-tests. A p value $<0.05$ was considered to be statistically significant. All analyses were performed using SAS (SAS Institute Inc., Cary, NC, U.S.).

\section{Results}

One hundred and four out of the 118 partial nephrectomy patients were reviewed (Table 1). The mean age was 62 years (standard deviation [SD] 13 years). The majority were male $(71 ; 68 \%)$ and a substantial proportion had comorbid disease, such as diabetes and hypertension (Table 1 ). The majority of surgery was performed using an open approach $(87 ; 84 \%)$ and none were performed robotically. Mean estimated blood 


\begin{tabular}{lc}
\hline \multicolumn{2}{l}{ Table 1. Patient and operative characteristics } \\
\hline \multicolumn{2}{l}{ Demographics } \\
\hline Patient characteristics \\
\hline Age (years) \\
Sex \\
Male \\
Female & $71(68 \%)$ \\
Diabetes & $33(32 \%)$ \\
Hypertension & $18(17 \%)$ \\
Heart disease & $58(56 \%)$ \\
Smoking history & $32(31 \%)$ \\
Kidney & $58(56 \%)$ \\
$\quad$ Right & \\
Left & $48(46 \%)$ \\
Estimated blood loss & $56(54 \%)$ \\
Change in creatinine 3 months & $346 \mathrm{cc}(\mathrm{SD} 587 \mathrm{cc})$ \\
postoperative & $5 \mu \mathrm{mol} / \mathrm{L}(\mathrm{SD} 3 \mu \mathrm{mol} / \mathrm{L})$ \\
\hline SD: standard deviation. & \\
\hline
\end{tabular}

loss was 346 cc (SD 587 cc). Mean change in creatinine three months postoperatively was $5 \mu \mathrm{mol} / \mathrm{L}$ (SD $3 \mu \mathrm{mol} / \mathrm{L}$ ).

\section{Clinical judgement scores and scoring systems}

The mean partial nephrectomy complexity score based on clinical judgement was 3.5 (SD 1.8) out of 10. There was strong agreement between surgeons in assessing tumour

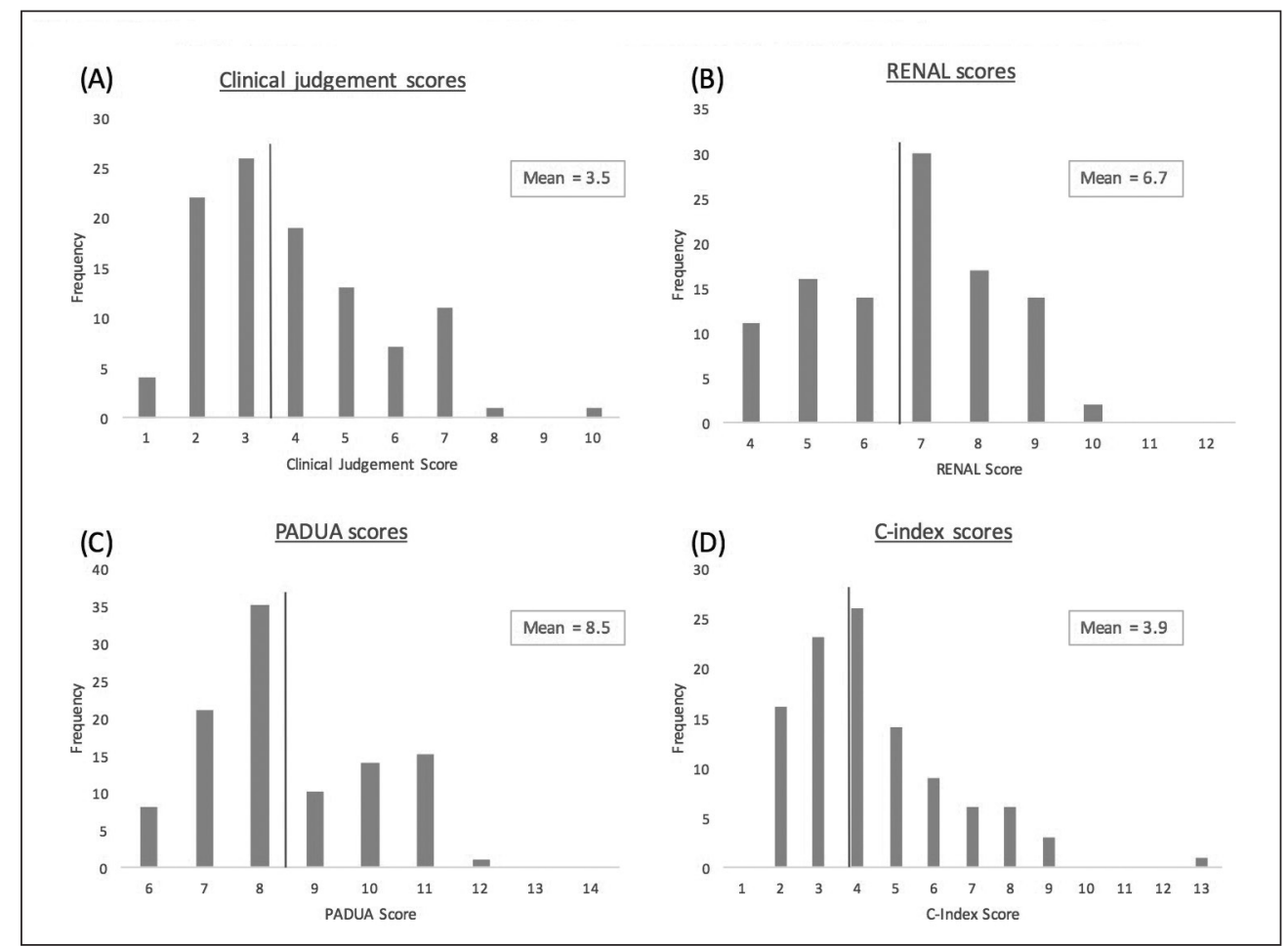

Fig. 1. Distribution of (A) clinical judgement scores; (B) RENAL scores; (C) PADUA scores; and (D) C-index scores. complexity (intraclass correlation coefficient $0.72 ; 95 \%$ confidence interval $[\mathrm{Cl}] 0.65,0.78)$. Clinical judgement scores were correlated to all three renal tumour scoring system scores (coefficients 0.53-0.6; $\mathrm{p}<0.001$ ). The mean RENAL score was 6.7 (SD 1.6) out of a maximum of 12, the mean PADUA score was 8.5 (SD 1.5) out of a maximum of 14, and the mean C-index score was 3.9 (SD 2). Histograms showing the distribution of clinical judgement scores and scoring systems are presented in Fig. 1.

\section{Ischemia time}

Mean ischemia time was 25 minutes (SD 10, range 5-65). The association between clinical judgement score, scoring system scores, and ischemia time were evaluated (Table 2). The correlation between clinical judgement scores and ischemia time was $0.27(p=0.005)$. The correlation between renal tumour scoring systems and ischemia time was 0.20 $(p=0.04)$ for RENAL, $0.21(p=0.03)$ for $\mathrm{C}$-index, and 0.26 $(p=0.007)$ for PADUA (Fig. 2).

\section{Postoperative complications}

In total, there were 75 postoperative complications, of which 30 were surgical and 45 were medical (Table 3). Of the surgical complications, four were Clavien-Dindo Grade 1, 14 were Grade 2, four were Grade 3A, four were Grade $3 \mathrm{~B}$, and four were Grade $4 \mathrm{~A}$. There were no Grade 5 complications. The most common surgical complication was requirement of blood transfusion. Of the medical complications, 14 were Grade 1, 27 were Grade 2, and four were Grade 4A. The most common medical complication was atelectasis.

Clinical judgement score was not statistically significantly associated with postoperative surgical complications $(p=0.16)$ or total complications $(p=0.17)$. RENAL and PADUA scores were significantly associated with surgical complications (RENAL $p=0.03$, PADUA $p=0.01$ ) and total complications (RENAL $p=0.03$, PADUA $p=0.01$ ). C-index score was not associated with incidence of postoperative surgical complications $(p=0.34)$ or total complications $(p=0.76)$. 
Kumar et al.

$\begin{aligned} & \text { Table 2. Associations between clinical judgement, tumour } \\
& \text { scores, and ischemia time }\end{aligned}$
\begin{tabular}{lcc}
\multicolumn{3}{l}{ Ischemia time } \\
\hline Variable & Coefficient $\left(\mathbf{r}^{2}\right)^{*}$ & p \\
\hline Clinical judgement & 0.27 & 0.005 \\
RENAL overall score & 0.2 & 0.04 \\
PADUA overall score & 0.26 & 0.007 \\
C-index score & 0.2 & 0.03 \\
\hline${ }^{*}{ }^{2}$ value is the proportion of variability predicted by clinical judgment or scoring system.
\end{tabular}

\section{Discussion}

Patients who receive partial nephrectomy have better preservation of renal function and similar risk of cancer recurrence compared to radical nephrectomy; $;^{1-4}$ however, partial nephrectomy patients are at higher risk of perioperative complications. Prior to renal tumour scoring systems, there were no accepted objective criteria available to stratify the complexity of tumour excision. As a result, it was not possible to easily compare cohorts across studies, nor was it possible to support individualized discussions with patients using evidence regarding the risks and benefits of the procedure. Previously, the reported benefits and risks of partial nephrectomy likely represented either an over or underestimation of the truth for a given patient.

In this study, we use ischemia time as a surrogate marker for tumour resection complexity, assuming that less complex cases should require less ischemia time to perform the tumour removal and kidney reconstruction compared to more complex cases. Our results show that overall, RENAL, PADUA, and C-index scores are associated with ischemia time. This is consistent with previously published reports. ${ }^{9}$ Our results also show that when urologists do not use a renal tumour scoring system, but simply review the preoperative axial imaging, they can also predict the complexity of a case as measured by ischemia time. This suggests that renal tumour scoring systems do not offer a significant advantage to the urologist at predicting the complexity of a partial nephrectomy.

Although RENAL, PADUA, and C-index scores might not be better than clinical judgement at predicting partial nephrectomy complexity, they still offer several advantages. Primarily, they provide a way of objectively describing a renal tumour. This allows for more meaningful comparisons to be made between clinical scenarios in the urological literature, the determination of eligibility criteria for clinical trials, and standardization of practice. Our results show that renal tumour scoring systems (RENAL and PADUA) predicted complications better than clinical judgement. In our study, however, the surgeons generated a clinical judgement score solely based on complexity of resection, not on the risk of complication.

Several limitations should be considered when interpreting this data. First, while the scores we evaluated are statistically associated with ischemia time, there remains a significant amount of variance in ischemia time that is not explained by either clinical judgement or renal tumour scoring systems. Therefore, although renal tumour scoring systems are no better than clinical judgment, neither of them is a good predictor of ischemia time. Only four surgeons were surveyed to create the clinical judgement scores. Furthermore, all four urologists have completed urological oncology fellowship training and perform a large volume of partial nephrectomies every year. A larger, more diverse group of urologists may not estimate case complexity in a similar manner. Another limitation is that clinical judgement scores were generated based on the complexity of performing an open partial nephrectomy, but $16 \%$ of cases were completed laparoscopically. For this minority of patients,

(D) C-index score. 


\begin{tabular}{lccc}
\hline $\begin{array}{l}\text { Table 3. Postoperative complications by Clavien-Dindo } \\
\text { grade }\end{array}$ \\
\hline Clavien-Dindo grade & \multicolumn{3}{l}{ Complications } \\
\cline { 2 - 4 } & Surgical & Medical & Total \\
\hline 1 & 4 & 14 & 18 \\
2 & 14 & 27 & 41 \\
3 & 8 & 0 & 8 \\
4 & 4 & 4 & 8 \\
5 & 0 & 0 & 0 \\
Total & 30 & 45 & 75 \\
\hline
\end{tabular}

the complexity rating may not be valid. Lastly, the use of renal ischemia time as a surrogate marker for complexity of surgical resection is imperfect, since there are other factors that impact renal ischemia time, including the skill and experience of the surgical trainee.

\section{Conclusion}

The majority of variance in ischemia time is not explained by clinical judgement or renal tumour scoring systems. In this study, renal tumour scoring systems were not better than the clinical judgement of urological oncologists at predicting ischemia time during partial nephrectomy. Although clinical judgement score was not statistically significantly associated with postoperative surgical complications or total complications, RENAL and PADUA scores were significantly associated with surgical complications. The ability of clinical judgement to predict more meaningful outcomes, such as perioperative complications, requires further study.

Competing interests: Dr. Cagiannos reports personal fees from Abbvie, Ferring, and Janssen, outside the submitted work. Dr. Lavallee reports grants from Sanofi and personal fees from Sanofi and Ferring, outside the submitted work. The remaining authors report no competing personal or financial interests.

This paper has been peer-reviewed.

\section{References}

1. Pierorazio PM, Johnson MH, Patel HD, et al. Management of renal masses and localizedrenal cancer: Systematic review and meta-analysis. J Urol 2016;196:989-99. https://doi.org/10.1016/i. juro.2016.04.081

2. Pierorazio PM, Patel HD, Feng T, et al. Robotic-assisted vs. traditional laparoscopic partial nephrectomy: Comparison of outcomes and evaluation of learning curve. Urology 2011;78:813-9. https://doi.org/10.1016/i.urology.2011.04.065

3. Poppel H, Da Pozzo L, Albrecht W, et al. A prospective randomized EORTC intergroup phase 3 study comparing the complications of elective nephron-sparing surgery and radical nephrectomy for low-stage renal cell carcinoma. Eur Urol 2007;51:1606-15. https://doi.org/10.1016/i.eururo.2006.11.013

4. Kutikov A, Uzzo RG. The RENAL nephrometry score: A comprehensive standardized system for quantitating renal tumour size, location, and depth. J Urol 2009;182:844-53. https://doi.org/10.1016/i. juro.2009.05.035

5. Ficarra V, Novara G, Secco $S$, et al. Preoperative aspects and dimensions used for an anatomical (PADUA) classification of renal tumours in patients who are candidates for nephron-sparing surgery. Eur Urol 2009;56:786-93. https://doi.org/10.1016/i.eururo.2009.07.040

6. Simmons MN, Ching CB, Samplaski MK, et al. Kidney tumour location measurement using the C-index method. J Urol 2010;183:1708-13. https://doi.org/10.1016/i.juro.2010.01.005

7. Lavallée LT, Desantis D, Kamal F, et al. The association between renal tumour scoring systems and ischemia time during open partial nephrectomy. Can Urol Assoc J 2013;7:E207-14. https://doi.org/10.5489/ cuaj.825

8. Canter D, Kutikov A, Manley B, et al. Utility of the RENAL nephrometry scoring system in objectifying treatment decision-making of the enhancing renal mass. Urology 2011;78:1089-94. https://doi.org/10.1016/i.urology.2011.04.035

9. Hayn MH, Schwaab T, Underwood W, et al. RENAL nephrometry score predicts surgical outcomes of laparoscopic partial nephrectomy. BJU Int 2011;108:876-81. https://doi.org/10.1111/i.1464410x.2010.09940.x

10. Okhunov Z, Rais-Bahrami $S$, George AK, et al. The comparison of three renal tumour scoring systems: C-index, PADUA, and RENAL nephrometry scores. J Endourol 2011;25:1921-4. https://doi.org/10.1089/end.2011.0301

11. Stroup SP, Palazzi K, Kopp RP, et al. RENAL nephrometry score is associated with operative approach for partial nephrectomy and urine leak. Urology 2012;80:151-6. https://doi.org/10.1016/i.urology.2012.04.026

12. Bruner $B$, Breau RH, Lohse $C M$, et al. Renal nephrometry score is associated with urine leak after partial nephrectomy. BJU Int 2011;108:67-72. https://doi.org/10.1111/i.1464-410X.2010.09837.x

13. Kapoor A. Renal tumour scoring systems: How useful are they? Can Urol Assoc J 2015;9:46-7. hitps://doi.org/10.5489/cuaj.2740

14. Dindo D, Demartines N, Clavien PA. Classification of surgical complications: A new proposal with evaluation in a cohort of 6336 patients and results of a survey. Ann Surg 2004;240:205-13. https://doi.org/10.1097/01.sla.0000133083.54934.ae

15. Patel HD, Ball MW, Cohen JE, et al. Morbidity of urological surgical procedures: An analysis of rates, risk factors, and outcomes. Urology 2015;85:552-60. https://doi.org/10.1016/j.urology.2014.11.034

Correspondence: Dr. Rodney H. Breau, Ottawa Hospital Research Institute, Division of Urology, Department of Surgery, University of Ottawa, OHtawa, ON, Canada; rbreau@toh.on.ca 